\title{
Rheumatic Fever \& Rheumatic Heart Disease: Azithromycin Must Replace Penicillin for Treatment and Prophylaxis
}

\author{
Lalchandani $A^{1}$, Senthiraj ${ }^{2}$, Godara $\mathrm{M}^{3}$, Singh $\mathrm{V}^{4}$, Kumar $\mathrm{A}^{5}$, Ranjan $\mathrm{A}^{6}$
}

\begin{abstract}
The standard and age-old treatment of RF/ RHD is a single injection of Benzathine Penicillin G given intramuscular after sensitivity test in a dose of 1.2 million units. For secondary prophylaxis, this is followed by Injection Benzathine Penicillin given intramuscular, each time after sensitivity test, after every 21 days (3weeks), in the same dose of 1.2 million units. The treatment and prophylaxis of RF/ RHD has never seriously been reviewed in the light of newer drugs discovered for GAS (Group A Streptococcus) after Penicillin. All the other drugs mentioned above are oral forms which could never be an alternative to Benzathine Penicillin due to the daily dose required, except for Azithromycin which has a long half-life and several other pharmacological properties which make it an ideal drug for treatment and prophylaxis of RF/ RHD. Benzathine Penicillin $\mathrm{G}$ is in use for past 60 years due to convenience of dosing, its undoubted efficacy in eradication of the GABHS, and the low cost. But the scene is changed now.
\end{abstract}

We must replace the use of Penicillin with a suitable drug, and never look back, for reasons detailed below.

Acute Rheumatic Fever (ARF) usually affects children aged 5 to 15 years, but recurrences of attacks with rheumatic activity or ongoing inflammation and intervals of rapid progression of valvular disease continue up to age 40 and even beyond. Rarely may it be detected for the first time even at the age of 50 years and beyond. ARF begins with a respiratory tract infection usually a sore throat caused by Group A Beta Haemolytic Streptococci (GABHS).

As there is no specific or definitive test for ARF, diagnosis is made by a combination of clinical features and to great extent the physician's discretion. For Rheumatic Heart Disease (RHD), the single most confirmatory evidence is the Echo Doppler Study. The standard and age-old treatment of RF/RHD is a single injection of Benzathine Penicillin G given intramuscular after sensitivity test in a dose of 1.2 million units. For secondary prophylaxis, this is followed by Injection Benzathine Penicillin given Intramuscular, each time after sensitivity test, after every 21 days (3weeks), in the same dose of 1.2 million units.

1. Dr. Arati Lalchandani

2. Dr. Senthiraj

3. Dr. M. Godara

4. Dr. V. Singh

5. Dr. A. Kumar

6. Dr. A. Ranjan

Address for correspondence:

Prof. Arati Lalchandani

7/154 Swaroop Nagar

Kanpur, U.P., India

Phone: +91-9839870077

E-mail: davelalchandani@gmail.com

\section{Prevention of Rheumatic Fever (RF)}

There is no treatment of ARF that has been proven to alter the likelihood of developing, or lessened the severity of RHD. In a study of 1790 patients from 11 countries, RF recurred in 8 $(.45 \%)$ in those who received Benzathine Penicillin prophylaxis compared with 11 of 96 (11.5\%) who did not comply. This shows that recurrence may occur even with best prophylaxis and that compliance is very important. So at best, our attempt should be to eradicate the GABHS infection at the outset and give maximum symptomatic relief possible to the patient.

The non-drug prevention of RF / RHD, like improving socio economic Conditions, housing, sanitation, overcrowding has not been possible in developing nations where RF / RHD is a major health problem. So the mainstay of primary prevention is timely and appropriate drug treatment.

The drug of choice for the past 60 odd years has been Penicillin in different forms namely: Phenoxymethyl Penicillin 500mg oral twice daily or Amoxycillin $1 \mathrm{~g}$ daily divided in 3 doses, Injection Procaine Penicillin IM twice a day, or Benzathine Penicillin G single dose IM 1.2 million units. Alternative to

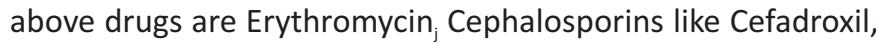
Cephalexin, Cefuroxime axetil, Cefpodoxime proxetil, Cefdinir, and Tetracyclines, Sulphisoxazole, Sulphadiazine, macrolides and Azalides etc. All above drugs have a big list of disadvantages of using in RF / RHD.

If this treatment is started within 9 days of onset of sore throat almost all cases of ARF will be prevented. This fact is extremely important if we want to shift to a better drug for primary prevention and also to treat recurrent attacks of ARF as well as for the secondary prevention in terms of safety, availability, compliance, efficacy, affordability and tolerability. The only drug with all these properties is Azithromycin. Ordinarily, sore throat whether viral or Streptococcal (GAS - Group A 
Streptococcus), resolves in 3 to 5 days but if we have to prevent $\mathrm{RF} / \mathrm{RHD}$, then all cases of sore throat even if cured or relieved of symptoms must be given the full course of Azithromycin $500 \mathrm{mg}$ $(12 \mathrm{mg} / \mathrm{Kg})$ once daily for 5 days $^{2}$.

\section{Secondary Prevention of RF}

Long term secondary prophylaxis is advocated in RF/RHD, as a known patient of $\mathrm{RF}$ is at higher risk for recurrent attacks of $\mathrm{RF}$ and progression of valvular heart disease than the general population. The best known antibiotic up till now has been Benzathine Penicillin G 1.2 million units given at interval of 3 to 4 weeks (with more data to support 3-weekly intervals). The treatment and prophylaxis of RF/ RHD has never seriously been reviewed in the light of newer drugs discovered for GAS (Group A Streptococcus) after Penicillin. All the other drugs mentioned above are oral forms which could never be an alternative to Benzathine Penicillin due to the daily dose required except for Azithromycin which has a long half-life and several other pharmacological properties which make it an ideal drug for treatment and prophylaxis of RF/ RHD. Benzathine Penicillin G is in use for past 60 years due to convenience of dosing, its undoubted efficacy in eradication of the GABHS, and the low cost. But the scene is changed now.

We must replace the use of Penicillin with a suitable drug, and never look back, for the following reasons:

$>$ Patients of RF/RHD are usually from the villages who first go to their family doctor or $\mathrm{CHC}$ for treatment. For the past two decades, with the gradually increasing distrust of the patients for the doctors and with the Consumer Protection Law for medical practice, the doctors, paramedics and the nurses have become very fearful of giving injections of Penicillin due to the severe fatal reactions which occur in more than 1 in 10,000 patients, which is not a small number by any means Considering that there may be more than 20 million people affected by RF/ RHD in the world today (AHA Scientific Statement Circulation March 24, 2009) $)^{3}$.

> Also, this drug Benzathine penicillin has become very difficult to procure and the patients have many a times to buy it in the black paying many times the cost of the drug.

$>$ They have to travel long distances in search of some person who will be ready to give them the injection, knowing the hazards. Even big hospitals, district hospitals and medical college hospitals refuse to give these injections due to the sudden severe reaction causing death of the patient or, they do so only after getting signed consents of the relative saying that they, are well aware and are prepared for the fact that their child (relation) may well drop down dead after the prick (even after-proper sensitivity test each time that the injection is administered).

$>$ We have come a long way from those days when there was no option apart from penicillin oral or injectable and it was a 'take or die' situation. Today when the patient's parents ask Doctor, is there any other option? - then one can't say no, that's the only drug if you want to save your child.

$>$ After an injection of Benzathine Penicillin, invariably the patient, usually a small child suffers from aches and pains all over and especially at the site of injection; fever, which does not recover any sooner than it's time for next injection.

$>$ The injection itself is oily and painful and more than $2 \mathrm{ml}$ in quantity. It has to be given deep intramuscular whereas the patients of RHO are usually from poor socioeconomic strata, very cachectic and malnourished with poor muscle mass.

> There are frequent cases of patients showing transient valvulitis of mitral and aortic valve after injection of Benzathine Penicillin. ${ }^{4}$ So ironically this becomes a cause for continuing' the injection for say another 5 years and then the patient ends up with the injection being prescribed lifelong. [May be this is the reason that there are recommendations for giving it lifelong].

$>$ Some drugs which interact with penicillin include warfarin, aspirin, tetracycline, diuretics etc. These are commonly coprescribed drugs in patients of RF / RHD.

> Benzathine penicillin is not for IV use at all. Inadvertent IV injection can cause cardiorespiratory arrest and death.

$>$ Hypersensitivity and allergic reactions, immediate and delayed are common.

$>$ It should not be injected near a nerve, artery or vein.

$>$ Pseudomembranous colitis is common with penicillin.

$>$ Clostridium difficile associated diarrhoea (COAD) occurs which can increase the morbidity and mortality.

$>$ There is enough evidence and experience with Benzathine Penicillin to show that it itself causes all features of ARF like fever, arthritis and even valvulitis, not to mention the well known fatal anaphylaxis risk ${ }^{4}$.

There is ample scientific supporting literature for all of the above.

All the above problems result in total noncompliance leading to the progression of valve destruction and death of the patient. Any amount of counseling also does not help as the patients and attendants are more terrified of the injection and its consequences compared to the pains and agony of the disease itself, which they realize will progress relentlessly sometimes irrespective of years of painful prophylaxis.

The solution is to give tablet Azithromycin $\mathbf{5 0 0 ~} \mathrm{mg}$ once daily for 5 days consecutively as initial treatment, and then 1 tablet of Azithromycin $500 \mathrm{mg}$ only once a week i.e. say every Sunday morning for 1 year only.

In our institution this antibiotic with more or less similar regime has been in use for 8 years and found to be nearly 100\% effective in terms of almost no relapse, recurrence or new cases of RF and RHD, nearly $100 \%$ compliance and freedom from side 
effects. It may be given for more than 1 year if at all there is sore throat or relapse of the disease i.e. any of the symptoms of disease or any suspicion thereof, for an extended period according to the discretion of the treating doctor.

This regime has been worked out with the following facts for backup and rationalization:

> The basis of giving Azithromycin only once a week is the long half-life of the drug which is sufficient to eradicate GAS, and suppress any autoimmune inflammatory process in the heart which may start mid-week, when the drug is given at weekend.

> Azithromycin is acid-stable and so can be taken orally without damage from gastric acids. It is readily absorbed, but its absorption is greater on an empty stomach. Azithromycin can be taken with or without food.

$>$ Time to peak concentration is 2.1 to 3.2 hours for oral dosage, and 1-2 hours for IV forms.

$>$ Due to high concentration in phagocytes Azithromycin is actively transported to site of infection. During active phagocytosis large concentration of Azithromycin is released.

$>$ The concentration of Azithromycin can be 50 times higher in the tissues than in plasma. This is due to ion-trapping and high lipid solubility of the drug. Concentrations in target tissues such as lungs and tonsils exceed the MIC 90 after a single dose of $500 \mathrm{mg}$. The single daily dose administration is a big advantage.

$>$ Azithromycin is the first of a class of antibiotics designated chemically as azalides. The chemical name of Azithromycin is 9-deoxy9-za9-a-methyl-9-

ahomoerythromycin.

$>$ The molecular weight is 749.0.

$>$ The mode of action of Azithromycin is inhibition of protein synthesis in bacteria by binding to 50 s ribosomal subunit and preventing translocation of peptides

$>$ Azithromycin's long half-life allows a large single dose to be administered and yet maintain bacteriostatic levels in the infected tissue for several days.

> Following a single $500 \mathrm{mg}$ dose, plasma concentration of Azithromycin declines in a polyphasic pattern with a mean apparent plasma clearance of $630 \mathrm{~mL} / \mathrm{min}$ and a terminal elimination half-life of 68 hours. The prolonged terminal half-life is thought to be due to extensive uptake and subsequent release of drug from tissues.

$>$ The drug persists in the tissues for 6 days if started with continuous 5 -days treatment.

$>$ Hence Azithromycin needs to be given only once a week as it has a long life of 6 days if given after loading dose for 5 days. Thereafter it can be given in a dose of 1 tablet weekly only. All other antibiotics need to be given daily, therefore Azithromycin is the best choice.

$>$ But each time, the drug must be started with 1 tablet of $500 \mathrm{mg}$ continuously for 5 days even when switching over from other antibiotics.

$>$ Azithromycin also has immune-suppressant properties.
> Azithromycin is freely available, safe in children and adults alike, cost effective, and has excellent anti-streptococcal activity.

> It is effective in complete eradication of streptococci from oropharynx and subsequent prevention of RF RHD. Caution-should be exercised when administered to patients with renal failure.

$>$ It can be safely given even in moderate hepatic impairment. In children it can be given as a single dose of $10 \mathrm{mg} / \mathrm{kg}$ or $20 \mathrm{mg} / \mathrm{kg}$ for 3 days only, with good efficacy. A daily dose of $500 \mathrm{mg}$ may be given to any child more than 5 years of age.

$>$ During pregnancy no evidence of harm to the fetus due to Azithromycin was found.

> Azithromycin was discovered in 1991 and is in constant use for GAS since then with excellent results. In a systematic review of 21 randomized controlled trials Azithromycin $20 \mathrm{mg} / \mathrm{kg} /$ day (3-day) achieved bacteriological eradication in $95 \%$ cases in acute streptococcal tonsillopharyngitis ${ }^{5}$.

In a study downloaded from http:|circ.ahajournals.org/ dec16, 2011,730 patients were given Azithromycin for ARF in dose of $500 \mathrm{mg}$ once a day for 5 days then $500 \mathrm{mg}$ on 2 consecutive days of the week i.e. only 2 days in a week. No patients showed relapse or rebound rheumatic activity or re infection with streptococci, there was no worsening of cardiac valve disease either. Compliance was $100 \%$. Patients who had no new problem attributable to RF/RHD were given Azithromycin prophylaxis for one year only. Thus it was shown that Azithromycin is very effective in treatment and prevention of RF/ RHD due to efficacy of treatment and high compliance ${ }^{6}$.

In a communication to Prof. A. Lalchandani, Dr. Stephen Marko of World Heart Federation, University of Connecticut has mentioned that there is great concern over the use of Benzathine Penicillin $G$ due to significant issues with quality and quantity of the drug with reports of shortages and poor quality penicillin. He echoes the sentiments that the treatment guidelines should be less stringent and according to the needs of the community. (Stephen.Marko@worldheart.org - Dec.6, 2011-survey based study on BPG).

According to an article in Paediatric Cardiology, 2010 August, from the Division of Cardiology, Department of Paediatrics of University of Virginia, a retrospective review of patients of ARF $<21$ years was performed. 144 patients of RF/RHD showed $n$ recurrence rate of $38 \%$, mean level of compliance with Benzathine penicillin was $59 \%$ in patients without recurrence of ARF, and $57 \%$ in patients with recurrence of ARF. This suggests that improved methods of primary and secondary antibiotic prophylaxis for ARF must be researched ${ }^{7}$.

The recurrence rate for $\mathrm{RF}$ in various studies is $3 \%$ to $8 \%$ over 5 6 years but rate of recurrence of RF in studies with 
Azithromycin was less than $3 \%$ consistently. A serum penicillin concentration of more than $0.02 \mu \mathrm{gm} / \mathrm{ml}$ is required to prevent recurrence ${ }^{8}$ but since Azithromycin persists in tissues in high concentrations therefore its efficacy is greater in preventing recurrences.

There is no rationale for giving RF / RHD prophylaxis for more than 1 year after an episode of sore throat, recurrence, relapse of evidence of progression of disease. If Azitrhomycin is started within 9 days of sore throat, it will prevent all cases of RF/RHD. Various studies have proved that it takes at least two weeks after a streptococcal infection / sore throat for autoimmune destruction leading to RF /RHD. Even in post streptococcal reactive arthritis valvular heart disease is seen only several months after the streptococcal infection in a small proportion of patients. These patients should receive secondary prophylaxis for up to 1 year after symptoms (Class Ilb, LOE CAHA Scientific Statement) ${ }^{3}$.

The duration of prophylaxis according to American Heart Association Recommendations is from 5 years after last attack to lifelong prophylaxis in patients with persistent valvular disease, with a footer that the recommendations be modified by individual circumstances as warranted ${ }^{8}$. It is very obvious from the footer that the duration of treatment recommended has been arbitrarily decided and not after serious deliberations over the strep infection, a possible time-interval for causing an autoimmune damage, its time gap for presenting as carditis or organic heart valve destruction, its persistence and disappearance from the blood stream, its susceptibility to the drug and comparison with other drugs.

Can any benefit be attributed to a drug which has to be taken lifelong and can it truly be called 'prophylaxis'. An ethical prescription should be based on the principle of 'greater benefit of the drug than the risk from its use' and what greater risk than death ..... as can occur with injection Benzathine Penicillin; and what greater benefit than $95 \%$ eradication of causative organism!

What are we waiting for? There cannot be a better, more effective drug for GAS than Azithromycin (Classl, LOE-C). Should we not all shift to Azithromycin for prophylaxis ? Can there be better proof than 8 years of consistent and exclusive use of this drug for treatment and prophylaxis of RF and RHD in our GSVM Medical College and associated hospitals catering to a huge urban and rural population in and around Kanpur districts where most of the patients are from lower socioeconomic strata and the incidence of RF/RHD is not less than 4 per thousand, still significantly high ${ }^{6,10}$. As we strongly considered the use of Benzathine. Penicillin injections unethical, dangerous, less effective and even harmful, with probability of fatal reactions, a direct comparison of Behzathine Penicillin and Azithromycin could not be done but in vitro comparison result show Azithromycin to be a superior drug for GABHS, the causative organism of RF/ RHD ${ }^{11}$.

The best safest and most effective treatment and prophylaxis of RF and RHD today is Tab Azithromycin $\mathbf{5 0 0 ~} \mathbf{m g}$ one daily for 5 days continuously followed by one tablet of Azithromycin $500 \mathrm{mg}$ once a week for one year only.

\section{REFERENCES}

1. International Rheumatic Fever Study Group M. Markowitz and E. Kaplan (USA); R. Cuttica (Argentina); X. Berrios (Chile); Z. Huang and X. Rao (People's Republic of China); P. L Wahi and H. K. Bali (India); D. Millard (Jamaica); J. Y. Choi and C. Y. Hong (Korea); H. A Majeed (Kuwait); P. Clarkson and J. Neutze (New Zealand), H. C. Lue (Taiwan); C. Vongprateep and C. Phornphutkul (Thailand), and S. Munoz (Venezuela).1991, Elsevier Ltd.

2. Shaantanu Donde, Mishra A, Kochhar P: Indian J Otolaryngology and Head Neck surgery, 7 Jan 2012:Azithromycin in Acute Bacterial URTI: An Indian Noninterventional Study.

3. AHA Scientific Statement: Michael A, Gerber, Chair Robert S. Baltimore MD, Charles B, Eaton; Michael Gewitz FAHA, Anne H. Rowley; Stanford T Shulman, Kathryn A Taubert : Prevention of Rheumatic Fever and Diagnosis and Treatment of Acute Streptococcal Pharyngitis. Circulation 2009 Mar 24;119(11):1541-51.

4. Thomson WO: Late Resident Medical Officer, Belvidere Infec Dis Hosp; Glasgow: Sudden Death Following injection Penicillin: British Medical Journal, July12, 1952.

5. Altamimi S, Khalil A, Khalaiwi KA, Milner R, Pusic MV, Al Othman MA (2009) Short versus standard duration antibiotic therapy for acute Streptococcal pharyngitis in children. Cochrane Database Systemic Review. Doi: 10.1002/146515858. CD004872. pub2

6. Arati Lalchandani, Mukesh Rana, Mayank Mehrotra, Prabhu K, Taruni Lalchandani : Benzathine Penicillin must be substituted with Azithromycin for treatment and prophylaxis of Rheumatic fever. Downloaded from http://circ.ahajournals.org/December $16,2011$.

7. Seckeler, Hoke TR, Gurka MJ, Barton LL : No demonstrable effect of benzathine penicillin on recurrence of rheumatic fever in pacific island population. Pediatric Cardiology. 2010 Aug; 31(6) : 849-52. E pub 2010 April. 22.

8. Harrison's Principles of Internal Medicine $18^{\text {th }}$ Edition, Ch 322, Acute Rheumatic Fever.

9. Daniels: ED, Mohanlal D, Pettifor JM: S Afr Med J.1994 Aug;84:477-81:RF prophylaxis in SA.

10. A Lalchandani, M Shameem, $S$ Chandra, P Sondhi, A Agarwal, $V$ Agarwal, P Neelam, G Preethi, U Pandey: Use of Alternative Drugs for Rheumatic Fever Prophylaxis in Place of Injection Benzathine Penicillin. Indian Heart Journal 2005; 57: 462.Abstract.

11. Schaad UB (2004) Acute Streptococcal tonsillopharyngitis : A review of clinical efficacy and bacteriological eradication. Journal of Internal Medicine 32: 1-13. 